PHARMACOTHERAPY

\section{Cystic fibrosis in ferrets}

Sun, X. et al. Sci. Transl. Med. 11, eaau7531 (2019)

Although evidence suggests that cystic fibrosis (CF) - a multiorgan disease caused by mutations in the gene encoding cystic fibrosis transmembrane conductance regulator (CFTR) - starts in utero, the long-term effects of in utero abnormalities on the pathology of CF remain unclear. VX-770 (ivacaftor) is a CFTR potentiator that rescues channel gating in dysfunctional CTFR proteins from patients carrying a $C F T R^{G 551 D}$ mutation. The results of a new study showing that in utero administration of VX-770 in CFTR ${ }^{G 551 D}$ ferrets protects the animals from developmental abnormalities and neonatal mortalilty associated with CF suggest that early interventions could improve the treatment of CF. The study also offers a new model to understand the role of CFTR in CF pathophysiology and inform future therapies.

https://doi.org/10.1038/s41684-019-0322-x

\section{STROKE}

\section{Cognitive impairment after stroke}

Cuartero, M.I. et al. J. Clin. Invest. 129,

1536-1550 (2019)

Although cognitive impairment is frequent in patients after a stroke, the mechanisms underlying post-stroke cognitive deficits remain uncertain. Animal models of the condition are lacking, which delays the understanding of its pathophysiology and the identification of therapies. A new study using mice subjected to middle cerebral artery occlusion (MCAO mice) to induce cortical ischemia suggests that the hippocampus largely contributes to long-term memory deficits after stroke. By showing that hippocampal neurogenesis after stroke positively correlates with memory impairment, that stroke-induced newborn neurons promote an aberrant hippocampal circuitry remodelling, and that inhibition of stroke-induced hippocampal neurogenesis mitigates hippocampusdependent memory deficits, the study supports the targeting of hippocampal neurogenesis as a potential approach for the treatment of cognitive impairment after stroke.

\section{BIOLOGICAL TECHNIQUES}

\section{Exosomes for DMD}

Rogers, R.G. et al. JCI Insight 4, e125754

Duchenne muscular dystrophy (DMD) is a genetic disease characterized by progressive muscle degeneration and weakness. DMD is caused by mutations in the DMD locus that alter the production of dystrophin, a structural protein connecting the internal cytoskeleton to the extracellular matrix in muscles. No cure exists for DMD and the use of anti-inflammatory steroids has been associated with severe side effects such as respiratory dysfunction. A new study reports that intravenous injection of cardiospherederived cells (CDCs), cardiac stromal cells with anti-inflammatory, antifibrotic and cardiomyogenic properties, improves the dystrophic phenotype of $m d x$ mice, a mouse model of DMD. The study also shows that CDCs improve the function and regenerative capacity of muscle by secreting exosomes, thereby identifying CDCs-derived exosomes as new therapeutic candidates for DMD.

https://doi.org/10.1038/s41684-019-0324-8

\section{GUT MICROBIOTA}

\section{Gut feeling}

Kraimi, N. et al. J. Exp. Biol. jeb.202879 (2019)

Several studies have started to link alterations in the gut microbiota (GM)-brain axis to gastrointestinal and neurological diseases. A study now suggests that the gut-brain axis also influences emotional reactivity. Germ-free chicks from a Japanese quail line (Coturnix japonica) selected for a high emotional reactivity $(E+)$ that had been colonized with GM from a line with low emotional reactivity (E-) showed reduced emotional behaviour compared with germ-free chicks from $\mathrm{E}+$ line colonized with GM from the $\mathrm{E}+$ line. Results were reversed when behaviour was tested at a later stage, most likely owing to the resilience of GM to recover its equilibrium present in the $\mathrm{E}+$ host. This study shows that GM transfer can influence emotional reactivity in Japanese quails and supports the existence of a GM-brain axis in birds.

https://doi.org/10.1038/s41684-019-0325-7

Alexandra Le Bras and Ellen P. Neff

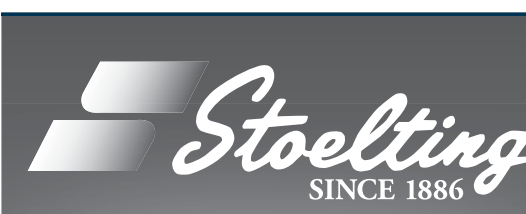

www.StoeltingCo.om

Lab Animal Identification (a) SMALL ANIMAL EARTAGS AND MARKERS
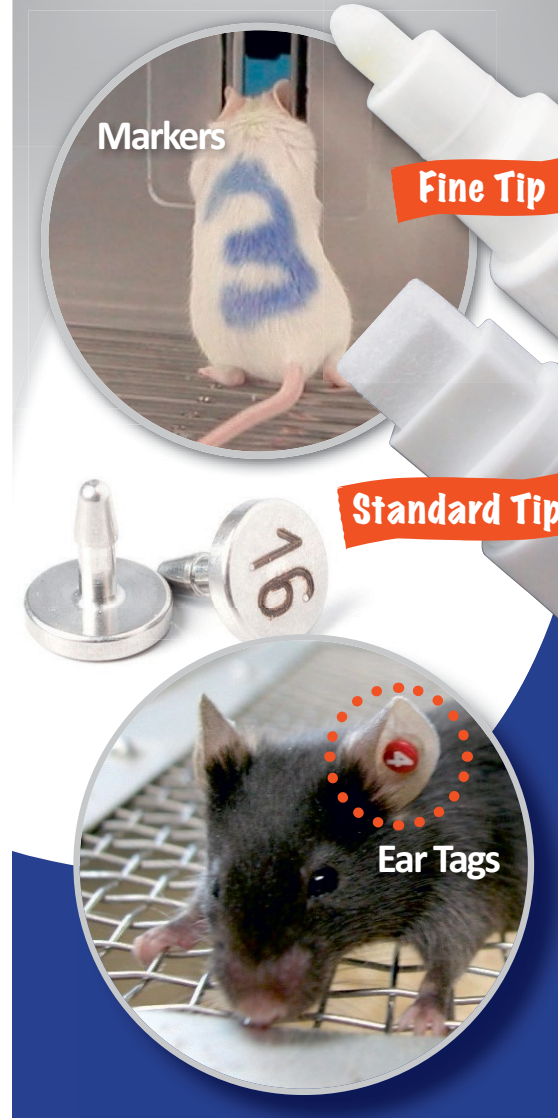

Ear Tag and Marker Features:

- Quick and easy identification

- Long-lasting

- Nontoxic, non-hazardous

- Multiple colors options available

Samples now available,

Contact Stoelting for

more details!

info@stoeltingco.com 